\title{
Priorización para el desarrollo de guías de práctica clínica en el Seguro Social de Salud del Perú (EsSalud): análisis multicriterio
}

\section{Prioritization for the development of clinical practice guidelines in the Seguro Social de Salud del Peru (EsSalud): multicriteria analysis}

\author{
Victor Suárez ${ }^{1, a}$, Carlos Alva-Díaz ${ }^{1,2,3, b}$, Raúl Timaná ${ }^{1, c}$, Patricia Pimentel ${ }^{1, d}$, Victor Dongo ${ }^{e}$ \\ Instituto de Evaluación de Tecnologías en Salud e Investigación del Seguro Social de Salud, EsSalud. Lima, Perú. \\ 2 Oficina de Apoyo a la Investigación y Docencia, Servicio de Neurología, Hospital Nacional Daniel Alcides Carrión. Callao, Perú. \\ 3 Red de Eficacia Clínica y Sanitaria. Lima, Perú. \\ a Médico infectólogo, Magister en salud pública. \\ b Médico neurólogo \\ c Médico gestor en salud \\ d Médico oncólogo \\ e Médico cirujano
}

An Fac med. 2018; 79(2):175-80 / http://dx.doi.org/10.15381/anales.v79i2.14946

Correspondencia:

Carlos Alva-Diaz

Calle Pedro Urraca 121, Condominio Up-

town, 1205-C, San Miguel. Lima, Perú.

Teléfono: +51 (1) 3409445

Correo electrónico:

carlos.alexander.alva@gmail.com

Recibido: 12 de junio2018

Aprobado: 27 de junio 2018

Conflictos de interés: Los autores

declaran no tener conflictos de interés

Fuentes de financiamiento: Instituto de Evaluación de Tecnologías en Salud e Investigación del Seguro Social de Salud, EsSalud. Lima, Perú

Contribuciones de autoría: Todos los autores han contribuido de forma sustancial al diseño, realización, análisis y presentación de los productos del presente artículo. Cada autor cree que este artículo representa la comunicación de un trabajo válido, verídico y ético, y cada autor ha revisado el contenido de este escrito y lo ha aprobado para su publicación. siendo esta autorización dictaminada por el autor de correspondencia, pero cuya responsabilidad es delegada a este, sin conflictos, por cada uno de los autores. Asimismo, los autores declaran que este trabajo nunca ha sido publicado, ya sea de forma parcial o total, en otra revista de divulgación de cientifica, y que el mismo no está siendo considerado en ninguna otra parte para su publicación.

Citar como: Suárez V, Alva-Díaz C, Timaná R, Pimentel $P$, Dongo V. Priorización para el desarrollo de guías de práctica clínica en el Seguro Social de Salud del Perú (EsSalud): análisis multicriterio. An Fac med. 2018:79(2):175-80.

DOI: http://dx.doi.org/10.15381/anales. v79i2.14946

\section{Resumen}

Objetivos. Describir el proceso de priorización para el desarrollo de guías de práctica clínica (GPC) en el Seguro Social de Salud del Perú (EsSalud). Métodos. El proceso de priorización se realizó mediante un análisis multicriterio en tres pasos: identificar las variables, obtener información de las mismas y asignarles una ponderación. Finalmente, se obtuvo una lista ordenada que fue puesta a consideración de los decisores de política. Resultados. Las cinco enfermedades con mayor número de AVISA (años de vida saludable perdidos) fueron: artrosis, glaucoma, esquizofrenia, osteoporosis y fracturas patológicas, y diabetes mellitus. Las de mayor gasto en medicamentos fueron: hemofilia, el grupo de nefritis, nefrosis (principalmente insuficiencia renal crónica), artritis reumatoide, cáncer de mama y SIDA. Las enfermedades prioritarias desde el punto de vista de la oferta de servicios fueron aquellas con alta mortalidad y alta discapacidad. Conclusiones. Se realizó el primer proceso de priorización de GPC en el Perú. Las 5 primeras enfermedades priorizadas fueron: artrosis, diabetes mellitus, glaucoma, enfermedad renal crónica y lumbago.

Palabras clave: Guias de práctica clínica; Enfermedad; Perú

\section{Abstract}

Objectives. To describe the prioritization process for developing the clinical practice guidelines (GPC) in the Seguro Social del Peru (EsSalud). Methods. The prioritization process was carried out through a multicriteria analysis in three steps: identify the variables, obtain information from them and assign them a weighting. Finally, an ordered list was obtained that was put to the consideration of the policy makers. Results. The five diseases with the highest number of DALY (disability adjusted life year) were osteoarthritis, glaucoma, schizophrenia, osteoporosis and pathological fractures, and diabetes mellitus. The most spending for drugs were hemophilia, the nephritis group, nephrosis (mainly chronic renal failure), rheumatoid arthritis, breast cancer and AIDS. Priority diseases from the point of view of the services offered were those with high mortality and high disability. Conclusions. This is the first process of guidelines prioritizing in Peru. The first 5 diseases prioritized were: osteoarthritis, diabetes mellitus, glaucoma, chronic kidney disease and lumbago.

Keywords: Practice guideline; Disease; Peru 


\section{INTRODUCCIÓN}

Las guías de práctica clínica son un instrumento útil para orientar a los profesionales de la salud en el manejo clínico de sus pacientes. Contribuyen a mejorar los resultados clínicos en salud, el uso racional de los recursos y reducen la variabilidad clínica ${ }^{1}$. La opinión general es que debemos pasar de guías basadas en opinión de expertos a guías basadas en la evidencia científica y que ofrezcan las mejores opciones de diagnóstico y tratamiento para los pacientes ${ }^{2}$. Para ello se han desarrollado metodologías para graduar la evidencia y elaborar las recomendaciones ${ }^{3}$, así como para adaptar guías basadas en evidencias a diferentes contextos $^{4}$. El Ministerio de Salud del Perú ha desarrollado también su propio documento metodológico para el desarrollo de guías, sea de novo o adaptación de guías ${ }^{5}$.

En este marco, uno de los primeros pasos es definir cuáles son las prioridades para la elaboración de guías de práctica clínica. Siendo este un proceso largo y complejo, demandante de recursos importantes, es necesario identificar cuáles son las prioridades para el uso racional de los recursos. El Instituto de Evaluación de Tecnologías en Salud e Investigación (IETSI) del Seguro Social de Salud del Perú (EsSalud), tiene como responsabilidad proponer las prioridades para la elaboración de las guías de práctica clínica a nivel institucional.

El presente artículo tiene como objetivo describir el proceso de priorización para el desarrollo de guías de práctica clínica (GPC) en el Seguro Social de Salud del Perú. Las prioridades que se definieran en este proceso serían las condiciones clínicas sobre las cuales a nivel institucional se tendrían que desarrollar las guías de práctica clínica. Posteriormente, en consenso con los especialistas, se determinaría cuál es el ámbito de las guías a desarrollar para cada condición clínica.

\section{MÉTODOS}

El Seguro Social de Salud del Perú tiene asegurados a 10, 557,997 personas a nivel nacional, lo cual representa la tercera parte de la población peruana. Entre el 2006 y el 2014, la población de asegurados aumento en un 63,9\%. En el año 2015, para establecer las prioridades de guías de práctica clínica se estableció mediante un análisis multicriterio un proceso de tres pasos. En el primer paso se evaluó cuáles serían las variables a tomar en cuenta para la priorización. En el segundo paso se valoró la lista de enfermedades con la información disponible de estas variables. Finalmente, se procedió al tercer paso que es la valoración final por los decisores de política de EsSalud.

Para el primer paso, consistente en seleccionar las variables, se revisó la literatura sobre experiencias similares y se consultó a expertos de la institución sobre variables que puedan ser de relevancia para la institución. Un criterio adicional para seleccionar las variables fue que hubiera información disponible. Así, se seleccionaron finalmente las variables de carga de enfermedad, gasto en medicamentos y las prioridades desde el punto de vista de la oferta de servicios.

En el segundo paso se buscó información con respecto a las variables seleccionadas. La carga de enfermedad fue estimada por la Oficina de Planeamiento e Inteligencia Sanitaria de EsSalud. La estimación disponible durante el proceso de priorización fue la correspondiente a la del año 2010. En la priorización se utilizó la metodología descrita por Murray, la cual es usada también por organismos internacionales y nacionales. La medición de carga de enfermedad proporcionó el número de años de vida saludables perdidos (AVISA) de la población asegurada por EsSalud. Los AVISA sintetizan los años de vida perdidos por muerte prematura y los años vividos con discapacidad.

El gasto en medicamentos se consideró con un indicador cercano a la medición del gasto en la atención de estas en- fermedades. Para evitar distorsiones que pudieran ocurrir en la compra de medicamentos de un año a otro, se consideró la suma del gasto realizado en los últimos tres años (2012 al 2014) en medicamentos. La lista de medicamentos se ordenó de mayor a menor según el volumen de gasto. Para cada medicamento se identificó cual es la indicación más frecuente en el cual es utilizado; esta indicación se asignó a una de las condiciones de la lista de enfermedades utilizada para el estudio de carga de enfermedad. Se excluyeron los medicamentos que tienen múltiples indicaciones como son el oxígeno, el cloruro de sodio y antibióticos. Si había más de un medicamento para la misma enfermedad, se sumaba el gasto. Si había un medicamento con dos a tres indicaciones, se repartía el gasto proporcionalmente de acuerdo a la frecuencia de presentación de las mismas, cuando la información estuviese disponible.

Las prioridades desde el punto de vista de la oferta de servicios de salud se obtuvieron del documento "Plan General de Salud 2015 - 2021 del Seguro Social de Salud-EsSalud", elaborado por la Gerencia Central de Prestaciones de Salud. En este documento se describen las condiciones de salud prioritarias en base a las cuales se elaboró el plan institucional a largo plazo.

A cada una de estas variables se les asigno un peso ponderado: 5 puntos a la carga de enfermedad, 3 al gasto en medicamentos y 2 a las prioridades desde la oferta de servicios de salud. Con carga de enfermedad se procedió a identificar la enfermedad con el mayor número de AVISA, a la cual se le asignó 5 puntos. A las demás enfermedades se les asignó un puntaje proporcional al número de AVISA teniendo como referencia el valor más alto de AVISA. El cálculo del puntaje a asignar a cada condición se hizo de la siguiente manera:
Número de AVISA de la enfermedad

Número de AVISA de la enfermedad con el valor más alto 
Con respecto al gasto en medicamentos se procedió de manera similar, asignando 3 puntos a la condición con más

gasto y luego proporcionalmente de acuerdo al gasto. El cálculo se hizo de la siguiente manera:

Puntaje asignado $=$

Gasto en soles de la enfermedad

Gasto en soles de la enfermedad con el valor más alto

Con respecto a las prioridades desde el punto de vista de la oferta de servicios, se asignó 2 puntos a las enfermedades que hubieran sido priorizadas en el plan mencionado y un punto a aquellas que no.

Teniendo la lista de enfermedades con los puntajes asignados se ordenó de manera decreciente según el puntaje total obtenido. Posteriormente se procedió al tercer paso que fue la valoración final por los decisores de política de EsSalud. Estos decisores fueron los gerentes de la Gerencia Central de Operaciones, la Gerencia Central de Prestaciones de Salud, y la Gerencia Central de Seguros y Prestaciones Económicas. Luego de la evaluación de ellos, la Presidencia Ejecutiva de EsSalud realizó una última valoración de las prioridades.

Tabla 1. Enfermedades en la población asegurada de EsSalud según el número de AVISA perdidos. EsSalud, Perú 2010.

\begin{tabular}{|c|c|c|c|}
\hline $\mathbf{N}^{\circ}$ & Enfermedades & AVISA & Puntaje \\
\hline 1 & Artrosis & 139400 & 5,0 \\
\hline 2 & Glaucoma & 92641 & 3,3 \\
\hline 3 & Esquizofrenia & 51717 & 1,9 \\
\hline 4 & Osteoporosis y fracturas patológicas & 46122 & 1,7 \\
\hline 5 & Diabetes mellitus & 44213 & 1,6 \\
\hline 6 & Enfermedad hipertensiva & 42047 & 1,5 \\
\hline 7 & Cataratas & 35814 & 1,3 \\
\hline 8 & Otras perinatales & 34196 & 1,2 \\
\hline 9 & Enfermedad de la neurona motora & 33039 & 1,2 \\
\hline 10 & Bajo peso/prematuridad & 31515 & 1,1 \\
\hline 11 & Anemia por déficit de hierro & 31054 & 1,1 \\
\hline 12 & Artritis reumatoide & 29761 & 1,1 \\
\hline 13 & Degeneración cerebral, demencia & 28308 & 1,0 \\
\hline 14 & Tumor maligno mama & 24368 & 0,9 \\
\hline 15 & Anoxia, asfixia, trauma al nacimiento & 22529 & 0,8 \\
\hline 16 & Nefritis, nefrosis & 20695 & 0,7 \\
\hline 17 & Enfermedad inflamatoria del corazón & 20135 & 0,7 \\
\hline 18 & Hipertrofia prostática & 19361 & 0,7 \\
\hline 19 & Enfermedad de Parkinson & 19164 & 0,7 \\
\hline 20 & Neumoconiosis/neumonitis & 17181 & 0,6 \\
\hline
\end{tabular}

Con respecto al gasto en medicamentos, se relacionaron los medicamentos con la lista de enfermedades para asignar un monto de gasto a cada una de ellas. Se utilizó la suma del gasto realizado durante el periodo 2012 - 2014 en nuevos soles. Este análisis se realizó con los primeros 57 medicamentos con mayor volumen de gasto y que se diferenciaban de manera importante con el resto de los medicamentos. Este grupo de medicamentos correspondía al $42 \%$ del volumen de gasto y son utilizados en 23 enfermedades de la lista. Estas fueron ordenadas de manera decreciente de acuerdo al gasto en nuevos soles y se les asignó un puntaje tal como se describió en la metodología. A las demás enfermedades se les asignó un puntaje similar al valor más bajo de las 23 enfermedades. Se observó que el grupo de enfermedades endocrinas y de la sangre fue el que tiene el mayor volumen de gasto en medicamentos. Dentro de este grupo, la principal enfermedad fue la hemofilia, cuyo gasto estuvo relacionado con el consumo de factor VIII, IX y VIla. Las otras enfermedades con mayor gasto en medicamentos fueron el grupo de nefritis, nefrosis (principalmente insuficiencia renal crónica), artritis reumatoide, cáncer de mama y SIDA. El detalle se puede observar en la tabla 2.

Con respecto a las prioridades, desde el punto de vista de la oferta de servicios de salud, estas fueron establecidas en el "Plan General de Salud 2015 - 2021 del Seguro Social de Salud - EsSalud". El mencionado documento tuvo en cuenta el diagnóstico situacional que realizó, el análisis de los determinantes de la salud, la situación de salud, el estudio de carga de enfermedad de EsSalud 2010 y el análisis de la oferta para definir sus prioridades. Estas sirvieron de orientación para la formulación del plan, lo cual supone una adecuación de la oferta de servicios a estas prioridades en el largo plazo. Las prioridades por oferta que se establecieron fueron las siguientes:

\section{Alta mortalidad}

Enfermedad cardiovascular: hipertensión arterial/miocardiopatías/accidentes cerebrovasculares/enfermedad reumática/enfermedad isquémica 
Tabla 2. Enfermedades en la población asegurada de EsSalud según el gasto en medicamentos. EsSalud, Perú 2012 - 2014.

\begin{tabular}{llcc}
$\mathbf{N}^{*}$ & Enfermedades & Gasto en soles & Puntaje \\
\hline 1 & Enfermedades endocrinas y de la sangre & 183970601 & 3,00 \\
2 & Nefritis, nefrosis & 140213330 & 2,29 \\
3 & Artritis reumatoide & 97596759 & 1,59 \\
4 & Tumor maligno mama & 80722446 & 1,32 \\
5 & SIDA & 48770891 & 0,80 \\
6 & Otras neuropsiquiátricas & 42182953 & 0,69 \\
7 & Diabetes mellitus & 28712117 & 0,47 \\
8 & Enfermedad hipertensiva & 25936879 & 0,42 \\
9 & Esquizofrenia & 25569674 & 0,42 \\
10 & Otras enf. órganos sentidos & 23588938 & 0,38 \\
11 & Linfoma, mieloma & 20942195 & 0,34 \\
12 & Úlcera péptica & 20682650 & 0,34 \\
13 & Otras nutricionales & 18259576 & 0,30 \\
14 & Tumor maligno riñón & 15757197 & 0,26 \\
15 & Otras infecciones & 13612653 & 0,22 \\
16 & Hepatitis B y C & 11060624 & 0,18 \\
17 & Leucemia & 10964844 & 0,18 \\
18 & Epilepsia & 10825551 & 0,18 \\
19 & Glaucoma & 10244099 & 0,17 \\
20 & Cataratas & 7211457 & 0,12 \\
\hline
\end{tabular}

- Tumores malignos: mama/cérvix/próstata/melanoma/leucemia/estómago

- Enfermedad genitourinaria: nefritis/nefrosis/hiperplasia benigna de próstata

- Enfermedad respiratoria: neumoconiosis

- Condiciones perinatales: bajo peso/prematuridad/asfixia nacer

- Diabetes mellitus

- Enfermedades del aparato digestivo: cirrosis/colecistitis/colelitiasis

- Anomalías congénitas cardiacas

2. Alta discapacidad

- Enfermedad osteomuscular y tejido conectivo: artrosis

- Neuropsiquiátricas: esquizofrenia

- Deficiencias nutricionales: anemia por deficiencia de hierro

Como ya se describió, se asignó 2 puntos a las enfermedades que se encontraban en esta lista de prioridades y un punto a aquellas que no.

Con los puntajes asignados a cada una de las tres variables, se procedió a sumar los mismos y a ordenar las enfermedades en forma decreciente de acuerdo al puntaje total obtenido. Las cinco enfermedades con mayor puntaje fueron: artrosis, nefritis y nefrosis, glaucoma, esquizofre- política para hacer los ajustes necesarios. En la reunión presencial participaron la Gerencia Central de Prestaciones de Salud, Gerencia central de Aseguramiento y Prestaciones Económicas, y Gerencia Central de Operaciones. Posteriormente la Presidencia Ejecutiva de EsSalud realizó la última evaluación de la propuesta. En la discusión se ratificó las condiciones identificadas a excepción de esquizofrenia. Se propuso incluir en la lista las condiciones de tumor maligno de cuello uterino y próstata, debido al incremento observado en los últimos años y la necesidad percibida de mejorar la oferta de servicios. De la misma manera, se propuso incluir también la condición de diabetes mellitus, dado que, si bien no estuvo entre las principales condiciones por carga de enfermedad, se encuentra relacionada directamente con las condiciones que sí lo están, como son la insuficiencia renal crónica y el glaucoma. Además de ello, también se propuso incluir la condición de lumbago debido a que es la principal causa de solicitud de incapacidad temporal y existe una alta variabilidad clínica en su manejo, especialmente en la definición de los días de descanso. Mortalidad materna también fue un tema que se decidió incluir pues es una
Definida la lista de enfermedades priorizadas, se convocó a los decisores de nia y tumor maligno de mama. En la tabla 3 se describen con detalle los puntajes obtenidos por cada enfermedad.

\section{Tabla 3. Enfermedades en la población asegurada de EsSalud según el puntaje} asignado para la priorización. EsSalud, Perú 2015.

\begin{tabular}{llcccc}
$\mathbf{N}^{\circ}$ & Enfermedades & $\begin{array}{c}\text { Carga de } \\
\text { enfermedad }\end{array}$ & Gasto & $\begin{array}{c}\text { Oferta de } \\
\text { servicios }\end{array}$ & $\begin{array}{c}\text { Puntaje } \\
\text { total }\end{array}$ \\
\hline 1 & Artrosis & 5,0 & 0,10 & 2,00 & 7,10 \\
2 & Nefritis, nefrosis & 0,7 & 2,29 & 2,00 & 4,99 \\
3 & Glaucoma & 3,3 & 0,17 & 1,00 & 4,47 \\
4 & Esquizofrenia & 1,9 & 0,42 & 2,00 & 4,32 \\
5 & Tumor maligno mama & 0,9 & 1,32 & 2,00 & 4,22 \\
6 & Enfermedades endocrinas y de la sangre & 0,1 & 3,00 & 1,00 & 4,10 \\
7 & Diabetes mellitus & 1,6 & 0,47 & 2,00 & 4,07 \\
8 & Enfermedad hipertensiva & 1,5 & 0,42 & 2,00 & 3,92 \\
9 & Artritis reumatoide & 1,1 & 1,59 & 1,00 & 3,69 \\
10 & Bajo peso/prematuridad & 1,1 & 0,10 & 2,00 & 3,20 \\
11 & Anemia por déficit de hierro & 1,1 & 0,10 & 2,00 & 3,20 \\
12 & Anoxia, asfixia, trauma al nacimiento & 0,8 & 0,10 & 2,00 & 2,90 \\
13 & Hipertrofia prostática & 0,7 & 0,10 & 2,00 & 2,80 \\
14 & Osteoporosis y fracturas patológicas & 1,7 & 0,10 & 1,00 & 2,80 \\
15 & Enfermedad cerebrovascular & 0,6 & 0,10 & 2,00 & 2,70 \\
16 & Neumoconiosis/neumonitis & 0,6 & 0,10 & 2,00 & 2,70 \\
17 & Tumor maligno cuello uterino & 0,4 & 0,10 & 2,00 & 2,50 \\
18 & Cirrosis & 0,4 & 0,10 & 2,00 & 2,50 \\
19 & Leucemia & 0,3 & 0,18 & 2,00 & 2,48 \\
\hline & Cataratas & 1,3 & 0,12 & 1,00 & 2,42 \\
\hline
\end{tabular}


condición relacionada con un indicador de impacto sanitario y cuya incidencia se está incrementando a nivel nacional en el presente año.

Cumplidas todas las etapas del proceso, las enfermedades priorizadas para la elaboración de guías de práctica clínica fueron:

- Artrosis
- Diabetes mellitus
- Glaucoma
- Enfermedad renal crónica
- $\quad$ Lumbago
- Mortalidad materna
- Tumor maligno de cuello uterino
- Tumor maligno de mama
- Tumor maligno de próstata

\section{DISCUSIÓN}

El presente artículo describe el proceso de priorización de las condiciones clínicas sobre las que se deben desarrollar las guías de práctica clínica en el Seguro Social de Salud, que atiende a la tercera parte de la población peruana. El proceso analizó las variables cuantitativas (carga de enfermedad, gasto en medicamentos, y prioridades establecidas de la oferta de servicios), así como una valoración cualitativa de los decisores de política. Esta última valoración adicionó prioridades que no habían sido reveladas en la primera medición. Las prioridades están enfocadas en enfermedades crónicas degenerativas. Tres de las nueve prioridades son una neoplasia y otras tres son enfermedades relacionadas entre sí (diabetes, glaucoma y enfermedad renal crónica).

La predominancia de las enfermedades crónicas degenerativas en la priorización para desarrollar guías de práctica clínica se explica por la situación epidemiológica del país. El Perú se encuentra en una dinámica transición epidemiológica en la que la mayor carga de enfermedad recae en este grupo de enfermedades, luego de años en los que el principal interés en salud publica eran las enfermedades transmisibles como la tuberculosis, la malaria y la infección por $\mathrm{VIH}^{6}$.

De las nueve prioridades finalmente identificadas, cinco fueron establecidas directamente por los decisores de política. Algunas de estas no se encontraban entre las primeras 20 condiciones consideradas para priorizar. La valoración de los decisores de política estuvo basada en variables que no fueron posibles medir de otra manera. La variabilidad clínica, la brecha entre la oferta y la demanda, y la identificación de problemas de salud pública de alto impacto social, fueron algunas de estas variables que complementaron la evaluación cuantitativa que se había realizado. De no haber considerado la opinión de los decisores de política no hubiéramos considerado varias condiciones clínicas en las que hay una necesidad sentida de desarrollar guías de práctica clínica.

Como afirman L. Reveiz y col., existe escasa evidencia sobre metodologías de priorización de temas clínicos para el desarrollo de GPC. La metodología de su desarrollo es compleja e incluye una serie de enfoques cuantitativos y cualitativos que reflejan las dificultades del proceso de priorización. Similar a nuestro estudio, en su metodología utilizaron dominios para priorizar a la carga de la enfermedad y al impacto económico, que nosotros medimos a través del gasto en medicamentos. Es importante señalar que en el estudio de L. Reveiz y col. los criterios y dominios utilizados para su priorizacion se agruparon en el tipo I, por el criterio de potenciales efectos sobre la salud, el tipo II, por los posibles efectos sobre costo y la viabilidad, el tipo III, por aspectos relevantes para la política sanitaria, siendo estos tipos similares a las variables utilizadas en nuestro estudio. Sin embargo, incluyeron otras variables como viabilidad y necesidad de información como dominios. Tambien consideraron otros dominios importantes como las preferencias del usuario, los eventos adversos potenciales para la promoción de la salud, y los efectos sociales, siendo estás posibles variables incorporadas por la valoración de los decisores de política que en nuestro estudio no fue posible medir?

Otros estudios han reportado el uso del método Delphi para establecer sus priorizaciones como el publicado por Loeffen y col., el cual tuvo como objetivo priorizar tópicos de cuidados de soporte en pacientes pediatricos oncológicos para el desarrollo de GPC en los paises bajos. El estudio finalmente estableció 10 tópicos prioritarios de 41 identificados inicialmente. Sin embargo, en el desarrollo de las mismas solo consideraron como actores a médicos especialistas sin incluir a otros actores como los decisores de política que sí participaron en nuestro estudio ${ }^{8}$. También, fue el método utilizado por M.T. Bayley y col. en su proceso de priorización para la elaboración de GPC sobre tópicos de traumatismo craneo encefálico, donde además del método Delphi modificado utilizaron un enfoque de investigación traslacional. Estos finalmente priorizaron 5 tópicos sobre traumatismo craneo encefálico de un grupo inicial de $66^{9}$.

H. Schunemann y col. afirman que, teniendo en cuenta los enormes recursos que se gastan en investigación y la importancia de proporcionar la mejor orientación a los responsables de las decisiones de atención médica, la asignación de fondos apropiados para síntesis de investigaciones y el desarrollo de guías basadas en la evidencia está justificada y, dada la complejidad del trabajo, se necesita colaborar para lograr la mejor y más rentable coordinación posible de estos esfuerzos ${ }^{10}$. Es en este proceso de priorización donde hay diferentes grupos de criterios a considerar, como los establecidos por la U.S. Institute of Medicine (IOM) que identifica 6 criterios generales: prevalencia, carga de la enfermedad, costos, variabilidad asistencial, potencialidad para mejorar los indicadores de salud, y reducción de costos. Tres de estas recomendaciones y criterios tambien fueron utlilizados en nuestro proceso de priorización. Sin embargo, también se proponen otros criterios en este proceso como son la incertidumbre o complejidad clínica, la disponibilidad de evidencias adecuadas y el estado del conocimiento, el interés médico, y el costo de desarrollo de la guía ${ }^{11}$.

El Instituto Nacional de Salud y Excelencia del Cuidado (NICE del ingles, National Institute for Health and Care Excellence), que es el organismo responsable de la elaboración de GPC en el Reino Unido, utilizó un proceso analítico jerarquizado (AHP, del ingles Analytic Hierarchy Process) para la priorización de los 
temas que debieran desarrollarse en $\mathrm{GPC}^{12}$. Se identificaron variables y se usaron técnicas cuantitativas y cualitiativas similares a lo realizado en nuestro estudio. En este proceso se identificaron variables consideradas en el proceso de priorización: 1) número de personas afectadas (población), medida mediante la carga de la enfermedad; 2) el costo de la enfermedad, sin embargo NICE utiliza indicadores de costo efectividad y en nuestro estudio se realizó mediante el gasto en medicamentos; 3) política, que en nuestro estudio se realizó mediante la identificación de prioridades desde el punto de vista de la oferta de servicios y la participación de los decisores de políticas en salud. NICE consideró otras variables que no fueron incorporadas en nuestro estudio: 1) la posibilidad de que la prácticas nocivas puedan ser modificadas, en términos de mejorar la equidad y acceso y; 2) disponibilidad de información relevante para contrarrestar posibles eventos o efectos adversos, o potenciales consecuencias nocivas de las practicas aplicadas.

La selección de las variables para la priorización en nuestro estudio tuvo como limitación la disponibilidad de información. Hubiera sido relevante tener información de enfermedades que afectan a poblaciones excluidas o especialmente vulnerables. No se contaba tampoco con información sobre la disponibilidad de guías de práctica clínica en los hospitales, ni mucho menos sobre la variabilidad clínica.

El país tiene diferentes regiones naturales, cada una con un perfil epidemiológico diferente. Por ejemplo, en la zona central del país hasta el $13,7 \%$ de la población puede tener serología positiva para cisticercosis ${ }^{13}$. Esta es una enfermedad de carácter regional y que afecta usualmente a poblaciones de bajos recursos. Una priorización de carácter nacional no podría incluir particularidades que son problemas de salud pública en determinadas regiones. Por ello, se hace necesario que a nivel regional se desarrollen procesos similares, de manera que abordemos problemas de salud que pueden no ser visibles desde la perspectiva nacional.

La ponderación que se dio a cada una de las tres variables evaluadas fue definida por el equipo investigador, y no mediante consulta a otros actores relevantes. El Seguro Social de Salud del Perú se financia exclusivamente por los aportes de sus asegurados y no tiene restricciones de cobertura prestacional, lo cual pone en riesgo la sostenibilidad finan- ciera de la institución, por ello la variable económica es muy relevante en cualquier priorización. Sin embargo, al momento de asignar la ponderación, no le dimos el mayor peso a esta variable pues consideramos como principal finalidad la de reducir la carga de enfermedad de la población.

La priorización presentada permitirá orientar los escasos recursos disponibles para el desarrollo de las guías de práctica clínica en el Seguro Social de Salud. La metodología seguida permitió considerar los principales problemas de salud de la población asegurada, debido a que hemos tomado en cuenta no solo variables medibles, sino también la opinión de los decisores de política quienes enriquecieron la evaluación con la consideración de otras variables solo factible de ser valoradas desde su perspectiva; dicho documento oficial de priorización institucional se encuentra publicado en la página web de EsSalud ${ }^{14}$. Para futuras priorizaciones es necesario contar con información más detallada de la carga de enfermedad por regiones y estratificada por poblaciones de riesgo o desatendidas. También es importante contar con estudios de los costos de la atención sanitaria de las principales enfermedades que afectan a la población asegurada. Asimismo, la información se debe complementar con los especialistas en las áreas priorizadas para definir los temas específicos de las GPC. La metodología seguida en esta priorización es objetiva, tienen un enfoque comprehensivo que recoge las variables más relevantes y es factible de ser reproducida en otros contextos. Este es uno de los pocos reportes disponibles en la literatura científica sobre un proceso de priorización para el desarrollo de guías de práctica clínica desde una perspectiva nacional.

\section{AGRADECIMIENTOS}

A la Dra. Virginia Baffigo, expresidenta ejecutiva del Seguro Social de Salud, EsSalud, por el apoyo necesario para la realización de este proceso de priorización.

\section{REFERENCIAS BIBLIOGRÁFICAS}

1. Woolf SH, Grol R, Hutchinson A, Eccles M, Grimshaw J. Clinical guidelines: potential benefits, limitations, and harms of clinical guidelines. BMJ. 1999 Feb 20;318(7182):527-30.

2. Attia A. Adaptation of international evidence based clinical practice guidelines: The ADAPTE process. Middle
EastFertil Soc J. 2013 Jun;18(2):123-6. DOI: https://doi org/10.1016/j.mefs.2013.03.002

3. Sanabria AJ, Rigau D, Rotaeche R, Selva A, MarzoCastillejo M, Alonso-Coello P. GRADE: Methodology for formulating and grading recommendations in clinical practice. Atencion Primaria Soc Esp Med Fam Comunitaria. 2015 Jan;47(1):48-55. DOI: 10.1016/j. aprim.2013.12.013

4. ADAPTE [Internet]. Manual and Recommendations for Use Feb 2010 Final.doc - adapte-resource-toolkitguideline-adaptation-2-0.pdf. [citado el 13 de febrero 2016]. Disponible en: http://www.g-i-n.net/documentstore/working-groups-documents/adaptation/adapteresource-toolkit-guideline-adaptation-2-0.pdf

5. Ministerio de Salud del Perú [Internet]. Documento Técnico: Metodologia para la elaboración de Guias de Práctica Clínica. [citado el 13 de febrero 2016]. Disponible en: http://www.minsa.gob.pe/dgsp/documentos/ Guias/M-GPC1.pdf

6. Velasquez A. La carga de enfermedad y lesiones en el Perú y las prioridades del plan esencial de aseguramiento universal. Rev Peru Med Exp Salud Publica. 2009;26(2):222-31.

7. Reveiz L, Tellez DR, Castillo JS, Mosquera PA, Torres $M$, Cuervo LG, et al. Prioritization strategies in clinical practice guidelines development: a pilot study. Health Res Policy Syst BioMed Cent. 2010;8:7. DOI: 10.1186/1478-4505-8-7

8. Loeffen EAH, Mulder RL, Kremer LCM, Michiels EMC, Abbink FCH, Ball LM, et al. Development of clinical practice guidelines for supportive care in childhood cancer-prioritization of topics using a Delphi approach. Support Care Cancer Off J Multinatl Assoc Support Care Cancer. 2015 Jul;23(7):1987-95. DOI: 10.1007/ s00520-014-2559-7

9. Bayley MT, Teasell RW, Wolfe DL, Gruen RL, Eng JJ, Ghajar J, et al. Where to build the bridge between evidence and practice?: results of an international workshop to prioritize knowledge translation activities in traumatic brain injury care. J Head Trauma Rehabil. 2014 Aug;29(4):268-76. DOI: 10.1097/ HTR.0000000000000053

10. Schünemann HJ, Woodhead M, Anzueto A, Buist AS, Macnee W, Rabe KF, et al. A guide to guidelines for professional societies and other developers of recommendations: introduction to integrating and coordinating efforts in COPD guideline development. An official ATS/ERS workshop report. Proc Am Thorac Soc. 2012 Dec;9(5):215-8. DOI: 10.1513/pats.201208-053ST

11. Atkins D, Perez-Padilla R, Macnee W, Buist AS, Cruz AA, ATS/ERS Ad Hoc Committee on Integrating and Coordinating Efforts in COPD Guideline Development. Priority setting in guideline development: article 2 in Integrating and coordinating efforts in COPD guideline development. An official ATS/ERS workshop report. Proc Am Thorac Soc. 2012 Dec;9(5):225-8. DOI: 0.1513/ pats.201208-055ST

12. Reddy BP, Kelly MP, Thokala P, Walters SJ, Duenas A. Prioritising public health guidance topics in the National Institute for Health and Care Excellence using the Analytic Hierarchy Process. Public Health. 2014 Oct;128(10):896-903. DOl:10.1016/j.puhe.2014.07.003

13. Jayashi CM, Arroyo G, Lightowlers MW, Garcia HH, Rodríguez S, Gonzalez AE. Seroprevalence and risk factors for Taenia solium cysticercosis in rural pigs of northern Peru. PLoS Negl Trop Dis. 2012;6(7):e1733. DOI: https://doi.org/10.1371/journal.pntd.0001733

14. EsSalud [Internet]. Guias de Práctica Clínica IETSI: Priorización de Guias de Práctica Clínica. Lima: EsSalud; 2015. [Fecha de acceso 30 de mayo de 2018]. Disponible en: http://www.essalud.gob.pe/ietsi/pdfs/guias/Priorizacion_de_guias_de_practica_clinica_en_Essalud.pdf 